\title{
Anthropometric and physical fitness profiles of Tunisian female soccer players: Associations with field position
}

\author{
Mohamed Moncef Kammoun ${ }^{1}$, Omar Trabelsi ${ }^{1}$, Adnene Gharbi ${ }^{1,2 *}$, Liwa Masmoudi ${ }^{1}$, Sleh \\ Ghorbel $^{1}$, Zouhair Tabka ${ }^{2}$, and Karim Chamari ${ }^{3}$
}

${ }^{1}$ High Institute of Sports and Physical Education, Sfax University, Sfax, Tunisia; ${ }^{2}$ Faculty of Medicine, Sousse University, Sousse, Tunisia; and ${ }^{3}$ Aspetar Orthopaedic and Sports Medicine Hospital, Doha, Qatar

Copyright: (C) 2020 M. M. Kammoun et al. This is an open access article licensed under the Creative Commons Attribution License (https://creativecommons.org/licenses/by/4.0/).

\begin{abstract}
Background: There is a lack of descriptive data available regarding the characteristics of female soccer players from the African continent or the Arab world. Objective: This study investigated the anthropometric and physical fitness profiles of Tunisian female soccer players in relation to field position. Methods: In all, 75 Tunisian sub-elite female soccer players, with an average of $4.3 \pm 1.6$ years of playing experience, including goalkeepers (GK; $n=11)$, central defenders ( $\mathrm{CD} ; n=13)$, external defenders (ED; $n=15)$, central midfielders $(\mathrm{CM} ; n=10)$, external midfielders (EM; $n=14$ ), and attackers (AT; $n=12$ ), underwent anthropometric evaluations (body height, body mass, and body fat percentage) and physical fitness tests (flexibility, countermovement jump [CMJ], 20-m sprint, and Yo-Yo intermittent recovery test level 1 [YoYoIR1]). Results: GK had significantly higher body mass $(67.3 \pm 2.4 \mathrm{~kg})$, body height $(169.3 \pm 1.7 \mathrm{~m})$ and fat percentage $(24 \pm 2.6 \%)$ compared to all field positions. AT were significantly faster $(3.64 \pm 0.10 \mathrm{~s})$ than GK $(3.75 \pm 0.09 \mathrm{~s})$ in the 20 -m sprint $(p<.01)$, but there were no significant differences among the other field positions. GK had a significantly lower Yo-YoIR1 performance $(625 \pm 84 \mathrm{~m})$ than other field position players $(p<.001)$, but no significant difference was found among other field positions. GK and AT showed significantly better CMJ performance (GK: $31.3 \pm 2.6 \mathrm{~cm}$, AT: $30.4 \pm 2.4 \mathrm{~cm}$ ) compared to other groups. GK had higher flexibility $(21.6 \pm 2.7 \mathrm{~cm}$ in the the sit-and-reach test) than all other positions $(p<.05)$, but no significant difference was observed among players in the field. Conclusions: Overall, we can conclude that, in a north African sample of female soccer players, anthropometric profiles vary by playing position. As well, positional differences can be found when testing physical fitness components. This information could help coaches design more effective training programs to achieve success in modern soccer.
\end{abstract}

Keywords: women football players, playing roles, flexibility, yo-yo field test, body fat percentage

\section{Introduction}

In recent years, participation in women's soccer has grown dramatically. In 2015, 30 million participants were recorded, which represents an increase of $37 \%$ compared to 2000 (FIFA, 2015). Nevertheless, comparisons of female and male players, especially in terms of physical fitness and anthropometric characteristics, are still quite limited. Previous studies have highlighted the need to develop specific physical abilities and anthropometric qualities in order to perform well in soccer. Specifically, aerobic endurance

\footnotetext{
* Address for correspondence: Adnene Gharbi, High Institute of Sport and Physical Education, Sfax University, Airport Road, Km 3,5, BP 384, 3000 Sfax, Tunisia. E-mail: adnenegharbi@yahoo.fr
}

(Castagna, Impellizzeri, Chamari, Carlomagno, \& Rampinini, 2006; Chamari et al., 2005; McMillan, Helgerud, Macdonald, \& Hoff, 2005; Stølen, Chamari, Castagna, \& Wisløff, 2005), the ability to repeatedly execute high-intensity actions (Little \& Williams, 2005), sprinting (Haugen, Tønnessen, Hem, Leirstein, \& Seiler, 2014; Little \& Williams, 2005), agility (Little \& Williams, 2005; Sporis, Jukic, Ostojic, \& Milanovic, 2009), flexibility, power (Arnason et al., 2004), body height, body mass, and body fat percentage (Almagia, Rodriguez, Barrraza, Lizana, \& Jorquera, 2008; Colquhoun \& Chad, 1986; Haugen et al., 2014; Hewitt, Withers, \& Lyons, 2008; Krustrup, Mohr, Ellingsgaard, \& Bangsbo, 2005) have all been identified as determinants of superior soccer performance in males and/or females. 
Even though information about physical fitness and anthropometric characteristics of female soccer players have been widely studied (Davis \& Brewer, 1993; Haugen et al., 2014; Jensen \& Larsson, 1992; Tumilty \& Darby, 1992), most conclusions tend to be relatively vague and are sometimes contradictory. For example, some researchers reported that aerobic power, sprinting ability, flexibility, muscle strength, height, body mass, and body fat percentage vary significantly from one playing position to another (Davis \& Brewer, 1993; Haugen et al., 2014; Jensen \& Larsson, 1992; Tumilty \& Darby, 1992), but other studies have yielded conflicting results, suggesting that there are no differences among positions (Hasegawa \& Kuzuhara, 2015; Nikolaidis, 2014; Sporiš, Čanaki, \& Barišić, 2007; Todd, Scott, \& Chisnall, 2013; Vescovi, Brown, \& Murray, 2006). Previous research that examined positional differences among female soccer players were limited by the small groups of players (Andersson, Randers, Heiner-Møller, Krustrup, \& Mohr, 2010; Hasegawa \& Kuzuhara, 2015; Ingebrigtsen, Dillern, \& Shalfawi, 2011; Krustrup et al., 2005; Lockie et al., 2018; Milanović, Sporiš, \& Trajković, 2012; Sporiš et al., 2007). Moreover, most of these studies evaluated physical fitness using laboratory tests performed on cycle ergometers (Gil, Gil, Ruiz, Irazusta, \& Irazusta, 2007; Hasegawa \& Kuzuhara, 2015; Ingebrigtsen et al., 2011; Krustrup et al., 2005; Nikolaidis, 2014), which is not specific to the sport of soccer. Indeed, testing related to soccer should be conducted on a soccer field in order to accurately evaluate specific activity patterns (Chamari et al., 2005; Stølen et al., 2005). Data regarding the anthropometric and physical fitness characteristics of female soccer players in most parts of Europe, America, and Asia are available (Andersson et al., 2010; Krustrup et al., 2005; Lockie et al., 2018; Thorpe \& Ebersole, 2008; Vescovi \& McGuigan, 2008), but, to the best of our knowledge, there is lack of studies investigating these characteristics of women's soccer players in North Africa. Hence, the main purpose of this study was to assess the anthropometric and physical fitness profiles of Tunisian female soccer players. We also aimed to investigate the likely physical and morphological variables related to the different positional roles in soccer. Thus, the hypothesis was that the anthropometric and physical fitness profiles of Tunisian female soccer players would differ from one playing position to another.

\section{Methods}

\section{Participants}

A total of 75 Tunisian female soccer players with an average of $4.3 \pm 1.6$ years of playing experience took part in the study. All players, from 8 different teams, were active in the first league and 13 of them were part of the national team at the time of the study: none of the players had ever participated in any international soccer competitions, so, for this level of Tunisian female soccer, we considered this sample of players to be sub-elite. The participants were classified into 6 groups according to their positional roles: goalkeepers (GK; $n=11$ ), central defenders (CD; $n=13$ ), external defenders (ED; $n=15)$, central midfielders (CM; $n=10)$, external midfielders (EM; $n=14)$, and attackers (AT; $n=12)$. All measurements were taken during 2 months of the competitive season (February and March 2016). During the study, players participated in an average of 4 to 5 weekly training sessions and 1 competitive match during each weekend with an average of $8.0 \pm 2.0$ hours per week. Test procedures were familiar to all participants. All players provided informed consent after verbal and written explanations of the experimental schedule and potential risks of the study were presented. The research protocol was approved by the institutional research ethics committee.

\section{Protocol}

All players completed a survey about their professional playing and training experiences and playing positions. In order to investigate the positional variables for different Tunisian female soccer profiles, all participants completed a series of anthropometric measurements (body height, body mass, and body fat percentage) and physical fitness tests (flexibility tests, countermovement jump [CMJ], sprint tests, and Yo-Yo intermittent recovery test [Yo-YoIR1]).

\section{Anthropometric measurements}

Height was measured to the nearest $0.1 \mathrm{~cm}$ by means of a stadiometer, and body mass was measured to the nearest $0.1 \mathrm{~kg}$ using analogue scales. Harpenden Skinfold Calipers were used to estimate body fat percentage using the 4-sites approach (triceps, biceps, subscapular, and suprailiac skinfolds) as mentioned by Durnin and Womersley (1974).

\section{Physical fitness tests}

To guarantee safe and appropriate preparation for the physical fitness tests, players participated in a uniform warm-up of approximately 15 minutes, which included running, sprinting, and multi-directional movements, as well as dynamic stretching exercises. The tests were completed in the following order, with 3 minutes of passive rest time between each test: flexibility test, CMJ, sprint test, and Yo-YoIR1. Each player was able to make 2 attempts per test (except the Yo-YoIR1). The best performance for each test was recorded for later 
analysis. Each participant was verbally encouraged to give her maximal effort during all tests.

\section{Flexibility}

The flexibility of the lower back and hamstring muscles was measured by the sit-and-reach test (Ayala, de Baranda, De Ste Croix, \& Santonja, 2012). Participants were instructed to sit on the floor with legs stretched out straight ahead and then reach forward as far as possible.

\section{CMJ test}

For the CMJ, each participant started from an upright standing position: the hands were placed on the hips throughout the test to eliminate any influence of arm swing. The participant rapidly squatted down until the knees were bent at approximately 90 degrees and then immediately jumped vertically as high as possible, landing on both feet at the same time. The height of the jump was investigated using an Optojump device (Microgate, Bolzano, Italy). Participants executed 3 jumping trials interspersed with 15 -second recovery periods, and the best one was recorded.

\section{Sprint test}

All participants performed 2 maximal speed trials over a 20-m distance from a static standing start. At the start, the foot was placed $30 \mathrm{~cm}$ behind the starting line. Two electronic photocells (Witty Wireless Training Timer, Microgate, Bolzano, Italy) were positioned at both the start $(0 \mathrm{~m})$ and finish $(20 \mathrm{~m})$ lines to record the time of the sprint to the nearest 0.1 seconds.

\section{Yo-YoIR1 test}

The Yo-YoIR1, as suggested by Krustrup et al. (2003), consists of a series of 20-m shuttle runs following a specific pace set by an audio metronome, interspersed with 5 -second rest intervals every $40 \mathrm{~m}$. The speed was progressively increased. If the female player did not reach the start line in time, a warning was given. The test was terminated when a participant received a second consecutive warning or when she dropped out because of fatigue. The total distance covered during the YoYoIR1 (including the two 40-m distances for which a player was warned) was calculated and recorded for further analysis.

\section{Statistical analysis}

All results are expressed as mean \pm standard deviation. The Kolmogorov-Smirnov test showed normal data distribution. One-way analysis of variance (ANOVA) was used to examine the differences among playing positions (GK, CD, ED, CM, EM, and AT). To estimate the strength of significant findings, effect size partial eta-squared $\left(\eta_{\mathrm{p}}^{2}\right)$ was used. Effect size values were interpreted as follows: $\eta_{\mathrm{p}}^{2} \geq .01$ corresponds to a small effect size, $\eta_{\mathrm{p}}^{2} \geq .06$ represents a medium effect size, and $\eta_{p}^{2} \geq .14$ is considered as a large effect size. Testretest reliability was expressed as the intra-class correlation coefficient $(I C C)$, and measurement error as a standard error of measurement (SEM). Fisher's post hoc tests were used for pairwise comparison. A $p$-value of less than .05 indicated statistical significance. All statistical analyses were conducted using STATISTICA Software (Version 13.3.0; TIBCO Software, Palo Alto, CA, USA).

\section{Results}

\section{Anthropometric measurements}

Concerning age and experience, the ANOVA demonstrated a significant effect for Position $(F(5,9)=2.83$, $\left.p=.022, \eta_{\mathrm{p}}^{2}=.17\right)$ and $(F(5,69)=2.71, p=.027$, $\left.\eta_{\mathrm{p}}^{2}=.164\right)$. In fact, GK were older and more experienced than the rest of player groups $(p<.05)$. However, no significant differences related to age or experience were observed among the players in other field positions (Table 1).

For body height and body mass, the ANOVA showed a significant effect for Position $(F(5,69)=8.54$, $\left.p<.001, \eta_{\mathrm{p}}^{2}=.382\right)$ and $(F(5,69)=4.53, p=.001$, $\left.\eta_{\mathrm{p}}^{2}=.247\right)$. GK had significantly higher body mass and body height. CD were taller than EM and ED $(p<.001)$ (Table 1).

As for body fat, the statistical analysis yielded a significant effect for Position $(F(5,69)=6.53$, $\left.p<.001, \eta_{\mathrm{p}}^{2}=.321\right)$. Outfield playing positions had lower body fat percentages than GK $(p<.01)$. On the other hand, no significant differences in body fat were observed among ED, CD, EM, CM, and AT (Table 1).

\section{Physical fitness tests}

In the 20-m sprint test, the ICC and SEM showed a very high reliability $(I C C=.98$ and absolute $S E M=0.01 \mathrm{~s})$. The ANOVA revealed a significant effect for Position $\left(F(5,69)=2.38, p=.047, \eta_{\mathrm{p}}^{2}=.134\right)$. AT were the fastest players and were significantly faster than GK $(p<.01)$. Yet, no significant differences were observed between AT and other playing positions (Table 2).

In the CMJ test, a high reliability was observed $(I C C=.88$ and absolute $S E M=1.9 \mathrm{~cm})$. As well, a significant effect for Position $(F(5,69)=7.61, p<$ $\left..001, \eta_{\mathrm{p}}^{2}=.355\right)$ was observed. GK and AT performed best on the CMJ $(p<.05)$. However, no significant differences were observed in CMJ among CM, EM, CD, and ED positions (Table 2). 

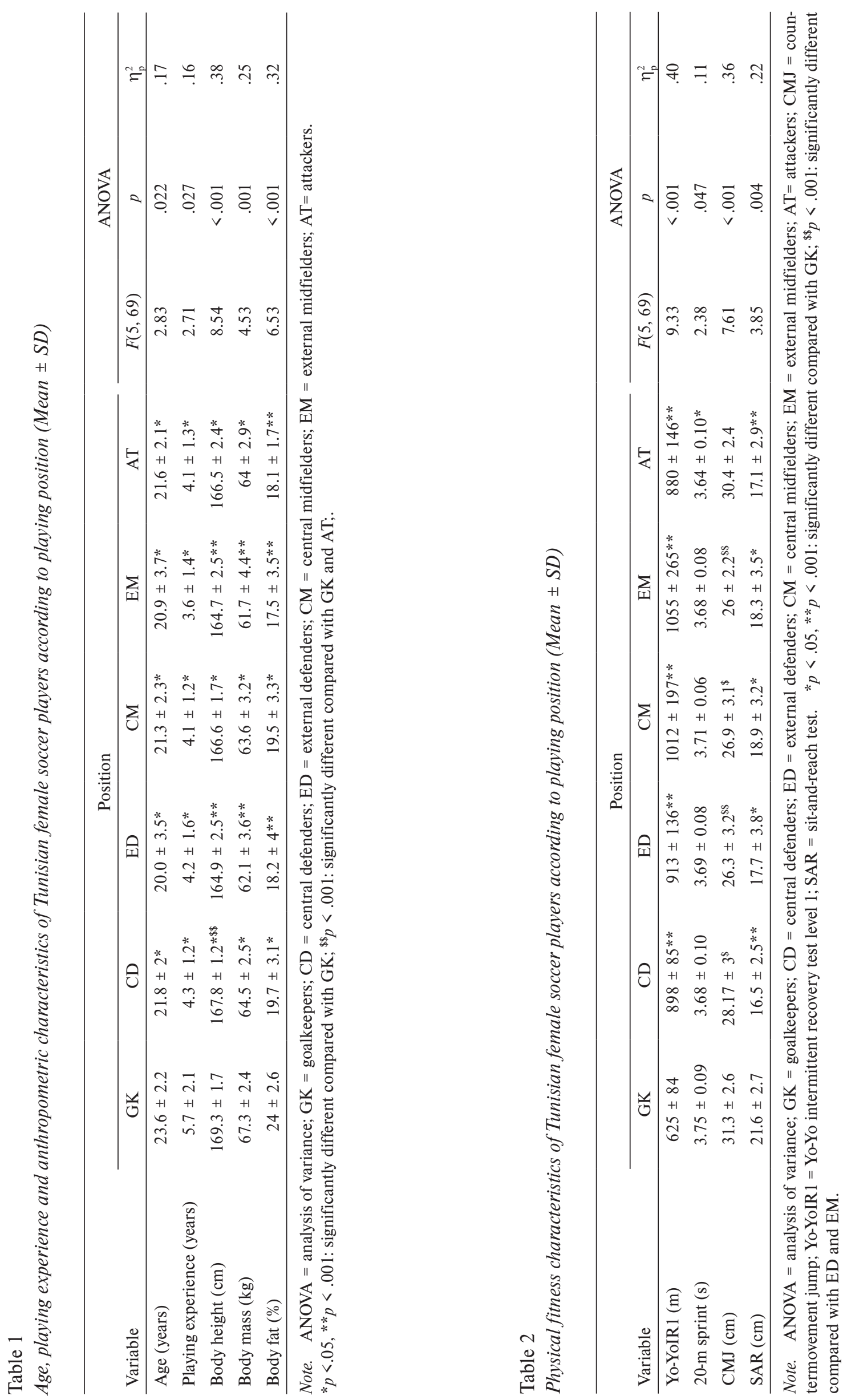
When about the sit-and reach test, the reliability was high $(I C C=.95)$, while absolute SEM showed low values $(0.74 \mathrm{~cm})$. The ANOVA demonstrated a significant effect for Position $(F(5,69)=2.38$, $\left.p=.047, \eta_{\mathrm{p}}^{2}=.134\right)$. GK had significantly greater flexibility than all other positions. Still, no significant differences were observed in flexibility levels among the other player groups (Table 2).

In the Yo-YoIR1 test, the ICC and SEM demonstrated very high reliability $(I C C=.96$ and absolute $S E M=1.9 \mathrm{~m})$. Moreover, a significant effect for Position $\left(F(5,69)=9.33, p<.001, \eta_{p}{ }^{2}=.403\right)$ was noted. The statistical analysis showed that GK demonstrated the lowest Yo-YoIR1 performance compared to all other positions $(p<.001)$. No significant differences in Yo-YoIR1 performance were observed among the other field player positions, although EM and CM had higher Yo-YoIR1 performance than other players (Table 2).

\section{Discussion}

Previous studies have evaluated the anthropometric and physical fitness profiles of female soccer players in most parts of America, Europe, and Asia. However, this is the first investigation that evaluated the characteristics of women soccer players from North Africa. The primary aim of the present study was to assess the anthropometric and physical fitness profiles of different player positions among Tunisian female soccer players. The present study revealed that GK were older and more experienced than players in all other positions. This contradicts previous results (Lockie et al., 2018; Sporiš et al., 2007; Vescovi et al., 2006), which concluded that there were no differences in age or playing experience among female soccer player positions. Female Tunisian GK reported having longer playing careers than outfield players. This may be explained by the facts that GK play a less physically demanding position than field players and that GK are less vulnerable to injuries (Reilly, 1994). The average age of female Tunisian GK was similar to that of the first league clubs in Greece (22.89 \pm 4.37 years) (Nikolaidis, 2014) but lower than that in Croatia (25.2 years). The average ages of Tunisian female players in other positions were similar to those reported from Norway and Greece (21 years; Haugen, Tønnessen, \& Seiler, 2012; Nikolaidis, 2014).

GK were the heaviest and the tallest players. CD were taller than EM and ED. These results were in accordance with previous studies (Campo et al., 2009; Haugen, Tønnessen, Hem, Leirstein, \& Seiler, 2012; Ingebrigtsen et al., 2011) that showed that GK are taller and heavier than midfielders and AT. Nevertheless, other studies concluded that height and body mass are similar for all playing positions (Can, Yilmaz, \& Erden, 2004; Vescovi et al., 2006). Analysis of the height and weight of females playing different positions at the most recent FIFA Women's World Cup (FIFA, 2015) revealed that $\mathrm{GK}$ were the tallest $(173.6 \pm 4.9 \mathrm{~cm})$ and heaviest $(67.0 \pm 5.2 \mathrm{~kg})$ players compared to players in all other positions. This is partly due to the role of GK, which requires a robust shape to defend their own goal during match play. Some anthropometric characteristics are thought to be beneficial for team strategies (Reilly, Bangsbo, \& Franks, 2000), and evidence suggests that players' anthropometric characteristics are related to their performances (Reilly et al., 2000). The average GK height in this study was lower than that of Norwegian players $(174.0 \pm 4.1 \mathrm{~cm}$; Haugen et al., 2014) but higher than that of Japanese collegiate women's soccer players $(161.7 \pm 6.2 \mathrm{~cm}$; Hasegawa $\&$ Kuzuhara, 2015). The mean heights of Tunisian female CM and EM were similar to American university female soccer players $(165.9 \pm 6.3 \mathrm{~cm}$; Vescovi et al., 2006).

The present study also showed that GK had significantly higher body fat percentage than all other positions. No significant differences in body fat percentage were observed among outfield playing positions. These results were very similar to those of Sporiš et al. (2007) and Nikolaidis (2014) who, using a similar technique, showed that GK have a higher body fat percentage than defenders, midfielders, and AT (Nikolaidis, 2014) and that there are no differences in body fat percentage among outfield playing positions (Nikolaidis, 2014; Sporiš et al., 2007). Nonetheless, the results of the present study conflict with those of Campo et al. (2009), who concluded that fullbacks registered the lowest body fat percentage. The difference obtained in our study is probably due to the differences in the players' competitive levels. The body fat percentage for GK reported in the present study was lower than that reported by Nikolaidis $(2014 ; 27.8 \pm 2.6 \%)$ but greater than that reported by Sporis et al. (2007; 13.7\%). The mean body fat percentages of Tunisian female ED $(18.2 \pm 4 \%)$ and EM $(17.5 \pm 3.5 \%)$ were higher than those reported by other authors, such as Sporiš et al. (2007), who measured a value of $14.3 \%$ among female Croatian AT, midfielders, and defenders.

Among physical performance variables, our results showed significant differences between AT and GK, but no significant differences between AT and other field positions. Our findings are in accordance with earlier findings for female Norwegian (Haugen et al., 2012) and English (Todd et al., 2013) players. However, other studies did not report any differences related to playing position among female college players (Hasegawa 
\& Kuzuhara, 2015; Lockie et al., 2018; Vescovi et al., 2006). When comparing our findings to previously published data within female soccer studies, the results of the sprint test in our study were lower than those reported by studies of Norwegian (Haugen et al., 2012) and Australian (Tumilty \& Darby, 1992) female international players. These differences could be, in part, explained by the variations in starting protocols. Using similar sprint test distances, participants may have had an advantage due to their positioning at the start of the sprints. The current study placed the participants 30 $\mathrm{cm}$ behind the first timing gate, while at $50 \mathrm{~cm}$ in other studies (Haugen et al., 2012; Tumilty \& Darby, 1992). However, the values found in our study for the 20-m test are similar to the values for Japanese collegiate women's soccer players (Hasegawa \& Kuzuhara, 2015).

In the present study, GK covered less distance in the Yo-YoIR1 than other position players $(p<.001)$. However, no significant difference was found among the other field positions. These findings were not in agreement with those of previous authors who have shown that individual differences in high-intensity running distances were linked to playing position; specifically, $\mathrm{CM}$ and $\mathrm{EM}$ run more than $\mathrm{AT}$ and $\mathrm{CD}$, thus reflecting differences in maximal oxygen uptake (Bradley et al., 2014). The lack of differences among various positions in our study could be explained by the absence of individualized training programs for female soccer players taking part in the study (Vescovi et al., 2006) and/or the level of play of the participants. Indeed, the lack of positional differences among field positions in Yo-YoIR1 performances observed in the present study is in accordance with the results obtained among female college players (Hasegawa \& Kuzuhara, 2015; Lockie et al., 2018) and English women's championship players (Todd et al., 2013) A comparison of our results with previously published data indicated that Yo-YoIR1 performance of GK was similar to that of Japanese collegiate women's soccer players (600 \pm 169 m; Hasegawa \& Kuzuhara, 2015), but lower than that of elite Serbian female soccer players $(780.0 \pm 84.8$ m; Milanović et al., 2012). Moreover, Yo-YoIR1 performances of players in field positions in the present study were below those of American collegiate women's soccer players (Lockie et al., 2018) and domestic and sub-elite players from Danish leagues (Bradley et al., 2014). Therefore, considering the present results, it is reasonable to qualify this sample of Tunisian female soccer players as sub-elite, since none of their physical performances reached elite standards. Still, this qualification is only based on their performances on physical fitness tests. Their level of play is out of the scope of the present study, and, according to the FIFA world rankings, it is clear that Tunisia is not ranked as one of the best countries for female soccer. (As of 2016, Tunisia was ranked $68^{\text {th }}$ worldwide, but the Tunisian team has qualified for the African Cup of Nations during the last decade.)

For the CMJ, GK and AT showed the best performance of all positions. However, there was no significant difference between CM, EM, and ED, despite a trend for $\mathrm{CD}$ to jump higher. These results do not agree with those of Vescovi et al. (2006), Haugen et al. (2012), Lockie et al. (2018), nor Hasegawa and Kuzuhara (2015). The higher CMJ observed in GK can be partly explained by the natural specialization of GK, who frequently perform vertical and lateral jumping actions during training and games. Previously reported CMJ results for GK and AT were $40.9 \pm 4.4 \mathrm{~cm}$ and $42.7 \pm 5.5 \mathrm{~cm}$, respectively, for college female soccer players (Vescovi et al., 2006), $29.1 \pm 3.3 \mathrm{~cm}$ and $25.7 \pm 4.5 \mathrm{~cm}$ for Japanese collegiate women's soccer players (Hasegawa \& Kuzuhara, 2015), $49.8 \pm 7.0 \mathrm{~cm}$ and $49.2 \pm 6.9 \mathrm{~cm}$ for English women's championship players (Todd et al., 2013), and $30.0 \pm 4.8 \mathrm{~cm}$ and $30.5 \pm 4.5 \mathrm{~cm}$ for junior female Norwegian players (Haugen et al., 2012). The mean values of the CMJ in Tunisian GK and AT were similar to elite Norwegian youth players (Haugen et al., 2012) but lower than English national standards (Todd et al., 2013).

When about flexibility, GK performed significantly better than all other positions. No significant differences were found among field positions. The present results are different from those of (Nikolaidis, 2014), who concluded that GK had lower flexibility values than defenders and midfielders. The flexibility of field players in the present study was lower than that reported by (Nikolaidis, 2014) but similar to that of GK in first league clubs in Greece (Nikolaidis, 2014). The higher flexibility observed in female Tunisian GK is probably due to the fact that, compared with other position players, a greater part of their training is devoted to developing flexibility.

\section{Conclusions}

In conclusion, the findings of this study suggested that, in the Tunisian first league of women's soccer, anthropometric profiles vary from one playing position to another. Exclusively, GK had exceptional physical characteristics that distinguished them from outfielders. As well, significant positional differences emerged when testing physical fitness components like cardiovascular endurance, acceleration, explosive lower-body power and flexibility. These findings related to the anthropometric and physical fitness profiles of female soccer 
players in Tunisia could be used by coaches to improve training programs and/or for selection purposes.

\section{Acknowledgments}

The authors wish to express their immense gratitude and appreciation to the English-native experts from BioMed Proofreading LLC who contributed to the editing of this manuscript.

\section{Conflict of interest}

There were no conflicts of interest.

\section{References}

Almagia, A. F., Rodriguez, R. F. J., Barrraza G. F. O., Lizana, P. J., \& Jorquera, A. C. A. (2008). Perfil antropométrico de jugadoras Chilenas de fútbol femenino [Anthropometric profile of female football - soccer Chilean players]. International Journal of Morphology, 26, 817-821.

Andersson, H. Å., Randers, M. B., Heiner-Møller, A., Krustrup, P., \& Mohr, M. (2010). Elite female soccer players perform more high-intensity running when playing in international games compared with domestic league games. Journal of Strength and Conditioning Research, 24, 912-919.

Arnason, A., Sigurdsson, S. B., Gudmundsson, A., Holme, I., Engebretsen, L., \& Bahr, R. (2004). Physical fitness, injuries, and team performance in soccer. Medicine \& Science in Sports \& Exercise, 36, 278-285.

Ayala, F., de Baranda, P. S., De Ste Croix, M., \& Santonja, F. (2012). Absolute reliability of five clinical tests for assessing hamstring flexibility in professional futsal players. Journal of Science and Medicine in Sport, 15, 142-147.

Bradley, P., Bendiksen, M., Dellal, A., Mohr, M., Wilkie, A., Datson, N., ... Bangsbo, J. (2014). The application of the Yo-Yo intermittent endurance level 2 test to elite female soccer populations. Scandinavian Journal of Medicine \& Science in Sports, 24, 43-54.

Campo, S. S., Vaeyens, R., Philippaerts, R. M., Redondo, J. C., de Benito, A. M., \& Cuadrado, G. (2009). Effects of lower-limb plyometric training on body composition, explosive strength, and kicking speed in female soccer players. Journal of Strength and Conditioning Research, 23, 1714-1722.

Can, F., Yilmaz, I., \& Erden, Z. (2004). Morphological characteristics and performance variables of women soccer players. Journal of Strength and Conditioning Research, 18, 480-485.

Castagna, C., Impellizzeri, F. M., Chamari, K., Carlomagno, D., \& Rampinini, E. (2006). Aerobic fitness and yo-yo continuous and intermittent tests performances in soccer players: A correlation study. Journal of Strength and Conditioning Research, 20, 320-325.
Chamari, K., Hachana, Y., Kaouech, F., Jeddi, R., MoussaChamari, I., \& Wisløff, U. (2005). Endurance training and testing with the ball in young elite soccer players. British Journal of Sports Medicine, 39, 24-28.

Colquhoun, D., \& Chad, K. E. (1986). Physiological characteristics of Australian female soccer players after a competitive season. Australian Journal of Science and Medicine in Sport, 18, 9-12.

Davis, J. A., \& Brewer, J. (1993). Applied physiology of female soccer players. Sports Medicine, 16, 180-189.

Durnin, J. V., \& Womersley, J. (1974). Body fat assessed from total body density and its estimation from skinfold thickness: Measurements on 481 men and women aged from 16 to 72 years. British Journal of Nutrition, 32, 77-97.

FIFA. (2015). FIFA Women's World Cup Canada 2015 TM. Technical report and statistics. Retrieved from https://resources. fifa.com/image/upload/fifa-women-s-world-cup-canada2015-technical-report-and-statistics-26708-2670891.pdf?cl oudid=jaeq2lvmczqjofxccj3u

Gil, S. M., Gil, J., Ruiz, F., Irazusta, A., \& Irazusta, J. (2007). Physiological and anthropometric characteristics of young soccer players according to their playing position: Relevance for the selection process. Journal of Strength and Conditioning Research, 21, 438-445.

Hasegawa, N., \& Kuzuhara, K. (2015). Physical characteristics of collegiate women's football players. Football Science, 12, 51-57.

Haugen, T. A., Tønnessen, E., Hem, E., Leirstein, S., \& Seiler, S. (2014). $\mathrm{VO}_{2 \max }$ characteristics of elite female soccer players, 1989-2007. International Journal of Sports Physiology and Performance, 9, 515-521.

Haugen, T. A., Tønnessen, E., \& Seiler, S. (2012). Speed and countermovement-jump characteristics of elite female soccer players, 1995-2010. International Journal of Sports Physiology and Performance, 7, 340-349.

Hewitt, A., Withers, R., \& Lyons, K. (2008). Match analyses of Australian international female soccer players using an athlete tracking device. In T. Reilly \& F. Korkusuz (Eds.), Science and football VI (pp. 224-228). Abingdon, United Kingdom: Taylor \& Francis.

Ingebrigtsen, J., Dillern, T., \& Shalfawi, S. A. (2011). Aerobic capacities and anthropometric characteristics of elite female soccer players. Journal of Strength and Conditioning Research, 25, 3352-3357.

Jensen, K., \& Larsson, B. (1992). Variations in physical capacity among the Danish national soccer team for women during a period of supplemental training. Journal of Sports Sciences, 10, 114-117.

Krustrup, P., Mohr, M., Amstrup, T., Rysgaard, T., Johansen, J., Steensberg, A., ... Bangsbo, J. (2003). The yo-yo intermittent recovery test: Physiological response, reliability, and validity. Medicine \& Science in Sports \& Exercise, 35, 697-705.

Krustrup, P., Mohr, M., Ellingsgaard, H., \& Bangsbo, J. (2005). Physical demands during an elite female soccer game: Importance of training status. Medicine \& Science in Sports \& Exercise, 37, 1242-1248.

Little, T., \& Williams, A. G. (2005). Specificity of acceleration, maximum speed and agility in professional soccer players. In T. Reilly, J. Cabri, \& D. Araùjo (Eds.), Science and football V: The proceedings of the Fifth World Congress 
on Sports Science and Football (pp. 289-296). London, United Kingdom. Routledge.

Lockie, R. G., Moreno, M. R., Lazar, A., Orjalo, A. J., Giuliano, D. V., Risso, F. G., ... Jalilvand, F. (2018). The physical and athletic performance characteristics of Division I collegiate female soccer players by position. Journal of Strength and Conditioning Research, 32, 334-343.

McMillan, K., Helgerud, J., Macdonald, R., \& Hoff, J. (2005). Physiological adaptations to soccer specific endurance training in professional youth soccer players. British Journal of Sports Medicine, 39, 273-277.

Milanović, Z., Sporiš, G., \& Trajković, N. (2012). Differences in body composite and physical match performance in female soccer players according to team position. Journal of Human Sport and Exercise, 7(Proc. 1), S67-S72.

Nikolaidis, P. T. (2014). Physical fitness in female soccer players by player position: A focus on anaerobic power. Human Movement, 15, 74-79.

Reilly, T. (1994). Physiological aspects of soccer. Biology of Sport, 11, 3-20.

Reilly, T., Bangsbo, J., \& Franks, A. (2000). Anthropometric and physiological predispositions for elite soccer. Journal of Sports Sciences, 18, 669-683.

Sporiš, G., Čanaki, M., \& Barišić, V. (2007). Morphological differences of elite Croatian female soccer players according to team position. Hrvatski Športskomedicinski Vjesnik, 22, 91-96.

Sporis, G., Jukic, I., Ostojic, S. M., \& Milanovic, D. (2009). Fitness profiling in soccer: Physical and physiologic characteristics of elite players. Journal of Strength and Conditioning Research, 23, 1947-1953.

Stølen, T., Chamari, K., Castagna, C., \& Wisløff, U. (2005). Physiology of soccer. Sports Medicine, 35, 501-536.

Thorpe, J. L., \& Ebersole, K. T. (2008). Unilateral balance performance in female collegiate soccer athletes. Journal of Strength and Conditioning Research, 22, 1429-1433.

Todd, M. K., Scott, D. S., \& Chisnall, P. J. (2013). Fitness characteristics of English female soccer players: An analysis by position and playing standard. In A. Murphy, T. Reilly, \& W. Spinks (Eds.), Science and football IV (pp. 374-381). Abingdon, United Kingdom: Routledge.

Tumilty, D., \& Darby, S. (1992). Physiological characteristics of Australian female soccer players. Journal of Sports Sciences, 10, 145.

Vescovi, J. D., Brown, T. D., \& Murray, T. M. (2006). Positional characteristics of physical performance in Division I college female soccer players. Journal of Sports Medicine and Physical Fitness, 46, 221.

Vescovi, J. D., \& McGuigan, M. R. (2008). Relationships between sprinting, agility, and jump ability in female athletes. Journal of Sports Sciences, 26, 97-107. 